EPJ Web of Conferences 101, 06019 (2015)

DOI: $10.1051 /$ epjconf/ 201510106019

(C) Owned by the authors, published by EDP Sciences, 2015

\title{
DIAMONDS: a new Bayesian nested sampling tool ${ }^{\star}$
}

\author{
Enrico Corsaro $^{\mathrm{a}}$ and Joris De Ridder
}

Instituut voor Sterrenkunde, KU Leuven, Celestijnenlaan 200D, B-3001 Leuven, Belgium

\begin{abstract}
In the context of high-quality asteroseismic data provided by the NASA Kepler Mission, we developed a new code, termed DIAMONDS (high-DImensional And multi-MOdal NesteD Sampling), for fast Bayesian parameter estimation and model comparison by means of the Nested Sampling Monte Carlo (NSMC) algorithm, an efficient and powerful method very suitable for high-dimensional problems (like the peak bagging analysis of solar-like oscillations) and multi-modal problems (i.e. problems that show multiple solutions). We applied the code to the peak bagging analysis of solar-like oscillations observed in a challenging F-type star. By means of DIAMONDS one is able to detect the different backgrounds in the power spectrum of the star (e.g. stellar granulation and faculae activity) and to understand whether one or two oscillation peaks can be identified or not. In addition, we demonstrate a novel approach to peak bagging based on multi-modality, which is able to reduce significantly the number of free parameters involved in the peak bagging model. This novel approach is therefore of great interest for possible future automatization of the entire analysis technique.
\end{abstract}

\section{The DIAMONDS code}

DIAMONDS is a software package developed in $\mathrm{C}++11$ and structured in classes in order to be as much flexible and configurable as possible [1]. It implements a more recent version of the NSMC algorithm [7,6,2,3]. The user can supply its own likelihoods, priors and models, according to the astrophysical problem of interest, by using a starting template. All the free parameters of the model and its corresponding Bayesian Evidence are therefore estimated by the code.

\section{Peak Bagging and Bayesian model comparison}

Determining how many different background signals are observed in the stars' power spectrum (Fig. 1, left) can be done by means of a model comparison based on the Bayesian Evidence, where each competing model includes a different representation of the background level. With the same method, one can also test the significance of an oscillation peak, in which the competing models will either include or not the peak to be assessed (Fig. 1, right). Model comparison becomes this way a very straightforward task [1].

\section{The novel approach: Multi-modality}

Conversely to other existing sampling methods (e.g. based on Markov Chain algorithm [5]) DIAMONDS allows to sample highly multi-modal distributions very efficiently [1]. The Eggbox function

* Software package available at the DIAMONDS code website: https://fys.kuleuven.be/ster/Software/Diamonds/.

a e-mail: emncorsaro@gmail.com

This is an Open Access article distributed under the terms of the Creative Commons Attribution License 4.0, which permits unrestricted use, distribution, and reproduction in any medium, provided the original work is properly cited. 

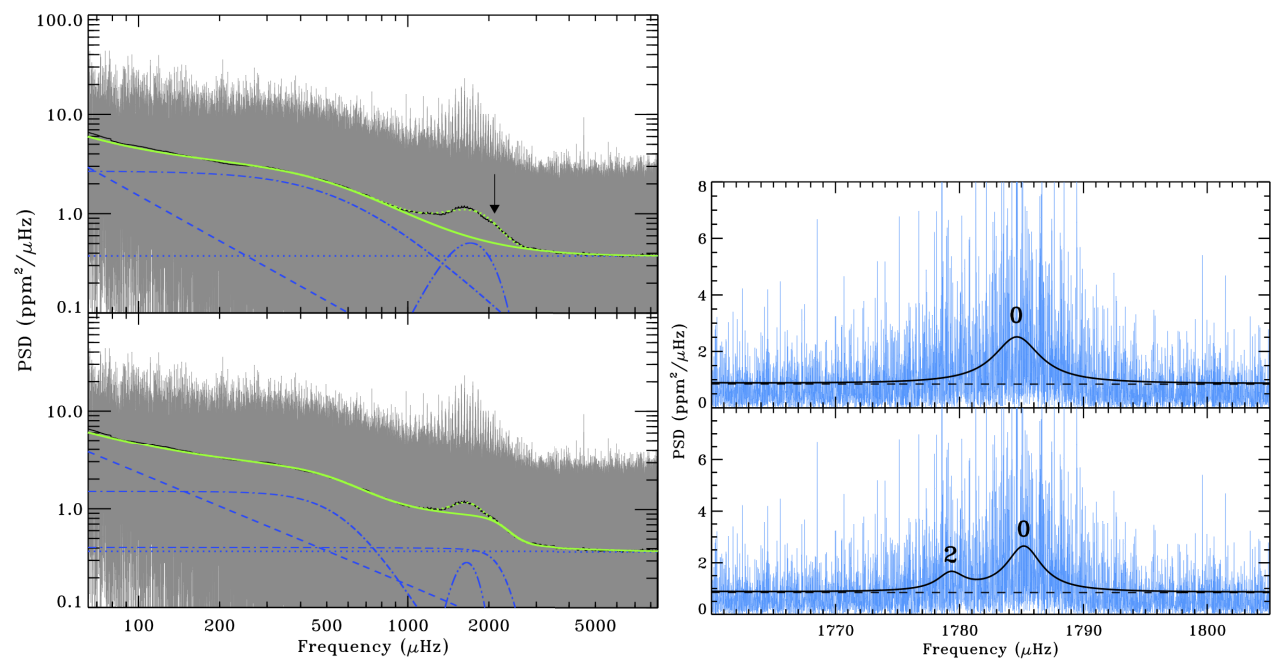

Fig. 1. Background fitting for two different models (left) indicated by the green line and an example of peak detection process (right) for the star KIC 9139163 calibrated following [4]. Model comparison favors the models used in the two bottom panels.

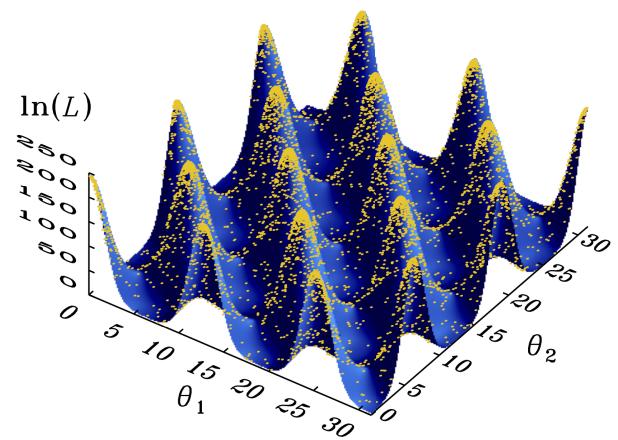

Fig. 2. Example of multi-modal distribution, the Eggbox function. Samples are shown by yellow dots.

(Fig. 2) is an example of degenerate (multiple) solution, namely a posterior probability distribution with several modes (hence multi-modal), sampled by DIAMONDS. We exploited the multi-modality as a novel approach to the peak bagging, succeeding in reducing the number of free parameters used to fit 27 consecutive oscillation peaks from 81 (a Lorentzian profile for each peak, hence 3 free parameters) to only 9 free parameters in total. The approach is very fast and efficient and is very well suited for automatizing the peak bagging analysis for future applications to several oscillating main sequence stars.

\section{References}

1. Corsaro E. \& De Ridder J., A\&A 571, 71 (2014)

2. Feroz, F. \& Hobson, M. P., MNRAS 384, 449 (2008)

3. Feroz, F., Hobson, M. P., \& Bridges, M., MNRAS 398, 1601 (2009)

4. García. R.A., Hekker, S., Stello, D., et al., MNRAS 414, L6 (2011)

5. Handberg, R. \& Campante, T. L., A\&A 527, 56 (2011)

6. Shaw, J. R., Bridges, M., \& Hobson, M. P., MNRAS 378, 1365 (2007)

7. Skilling, J., AIP Conference Proceedings 735, 395 (2004) 